\title{
POLYCICLIC AROMATIC HYDROCARBON IN URBAN AEROSOL
}

\author{
M.G. PERRONE ${ }^{1}$, E.BOLZACCHINI ${ }^{1}$, V.GIANELLE ${ }^{2}$, G. MOGNASCHI ${ }^{2}$ \\ ${ }^{1}$ Department of Environmental Science, University of Milano-Bicocca, Piazza della Scienza 1, \\ 20126 Milano, Italy \\ ${ }^{2}$ ARPA Lombardia - Milano Città, Via Juvara 22, 20129 Milano, Italy
}

Keywords: PAHs, urban aerosol, traffic source

\begin{abstract}
INTRODUCTION
Polyciclic aromatic hydrocarbons (PAHs) are produced by high-temperature reactions such as incomplete combustion and pyrolysis of fossil fuels and other organic materials. Studies have estimated that mobile sources are the major contributors to PAHs content of urban and suburban aerosol (Nielsen T., 1996).

High concentrations of $\mathrm{PM}_{10}$ are associated to adverse health effects and PAHs, primarily adsorbed to fine particles, account for most of the mutagenic potency that can be assigned to specific compounds (Hanningan et al., 1998).

PAHs can be found both in the gas and in the particulate phase, and for more volatile PAHs this is a function of temperature: PAHs with a higher molecular weight, which are more toxic, are always primarily associated with particulate phase (e.g B[a]P more than $90 \%$ in the particulate phase) (Odabasi M., 1999)
\end{abstract}

\section{METHODS}

PM10 and PM2,5 daily concentrations are constantly monitored in Milan using a low-volume filter system (flow rate of $16,67 \mathrm{l} / \mathrm{min}$ and a sampling time of $24 \mathrm{~h}$ ). The sampling is done $3 \mathrm{~m}$ above ground, in a site (Messina Street, Milan) close to the city centre, not affected to point sources and therefore well representative of background pollution levels for the urban area.

After sampling, filters were preserved in the darkness at $-20^{\circ} \mathrm{C}$ (avoiding photo degradation and evaporation losses) and some of them were analysed for PAHs. Since July 2001 to March 2003 about 200 samples of PM2,5 and PM10 were analysed for PAHs from all seasons to study pollution level and concentration trend. A method for the routine analysis of PAHs in atmospheric particulate samples has been developed. PAHs are extracted from the air particulates retained in the filters through ultrasonication. Different organic solvents (acetonitrile, acetone:esano and dichloromethane) and ultrasonication times were tested. Good results (recoveries for analysed PAHs $>70 \%$ ) were achieved by performing filter extraction with $2 \mathrm{ml}$ of acetonitrile in a very short time ( $3 \mathrm{~min}$ ), by using an ultrasonic bath specifically developed to maximize the efficiency of extraction from particulate matter.

Determination of PAHs in atmospheric particulate matter requires high sensitivity and high resolution and selectivity (due to the complexity of the matrices: presence along with PAHs of many interfering substances). Two different analytical techniques, high performance liquid chromatograpy (HPLC) with fluorescence detector and gas chromatograpy (GC) with mass spectrometric (MS) detection, were used.

From an intercomparison test it resulted that measurements of PAHs in particulate samples ( $\mathrm{n}=21$ : each sample extracted and then divided in two aliquots) are in good agreement for the two analytical techniques $\left(\mathrm{R}^{2}>0,9\right)$ but, to obtain such good results, it is necessary to correct for interfering species using reference standards which reproduce the complexity of the matrix. All data were corrected according to certificated values of the reference standard NIST 1649a.

\section{CONCLUSIONS}

In this study concentrations for 8 out of the 16 EPA PAH priority pollutants and benzo[e]pyrene are presented and discussed for PM samples. Only PAHs with higher molecular weight, which are primarily in the particulate phase, were considered (benzo[a]anthracene, crysene, benzo[b]fluoranthene, benzo[k]fluoranthene, benzo[a]pyrene, dibenzo[a,h]anthracene, indeno[1,2,3-cd]pyrene, benzo[ghi]perylene).

PAHs show a strong seasonal trend and, during winter, concentrations are about 100 times higher than summer. In winter concentration levels for analysed PAHs reached $70 \mathrm{ng} / \mathrm{m}^{3}(\Sigma$ PAHs $)$, while in summer typical values are under $1 \mathrm{ng} / \mathrm{m}^{3}$. 


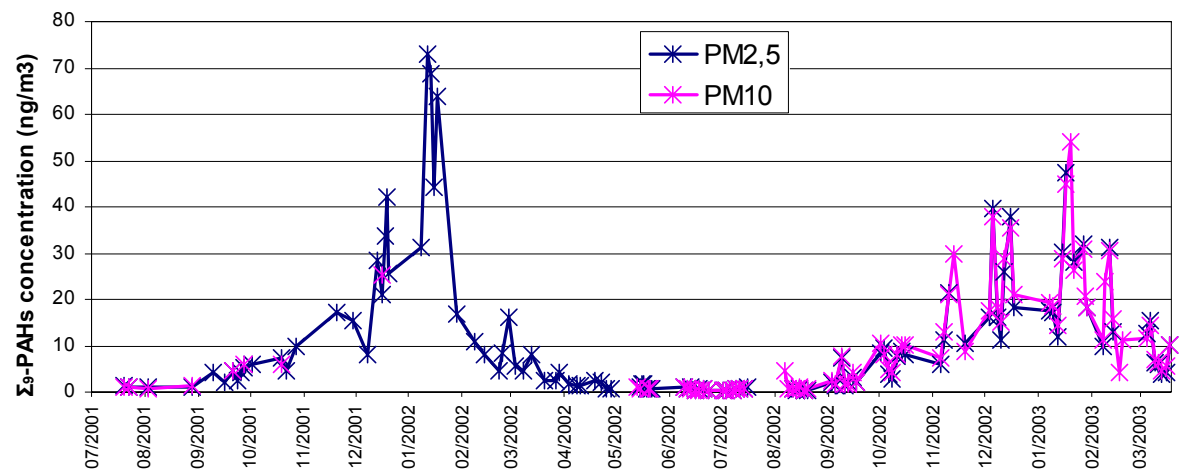

Figure 1. PPAHs concentration $\left(\mathrm{ng} / \mathrm{m}^{3}\right)$ in PM10 and PM2,5 daily samples for the urban area of Milan (Messina street)

PAHs are quite completely associated to fine particles. In figure 1 daily concentration of $\Sigma \mathrm{PAHs}$ is reported both for PM2,5 and PM10 samples: PAHs are more than 90\% in PM2,5 fraction.

PAHs size distribution in atmospheric particles was better studied using an high volume six stage cascade impactor (Staplex, $1,3 \mathrm{~m}^{3} / \mathrm{min}$ ). An unimodal distribution was found, and the peak is for the finest fraction collected: more than $70 \%$ of particulate $\mathrm{PAH}$ is adsorbed onto particles less than $0,5 \mu \mathrm{m}$ in diameter.

PAHs are a very reduced fraction of PM (less than $0,06 \%$ of $\mathrm{PM}_{2,5}$ ), but this percentage changes with seasons: during winter PM is richer in PAHs (a factor of 10), that is, for the same PM concentration, its potential toxicological effect is higher.

In urban atmosphere, high PAHs concentrations are primarily due to road traffic. During 3 days (winter 2001), 4h samples of PM2,5 have been collected and analysed for PAHs. Trends have been compared with traffic: maximum content of PAHs in PM2,5 is during hours (8.00-12.00) with maximum of heavy traffic (vehicles longer than $7 \mathrm{~m}$ ). Even if heavy traffic is less than $10 \%$ of total traffic, it seems to be a significant source of PAHs in the urban atmosphere.

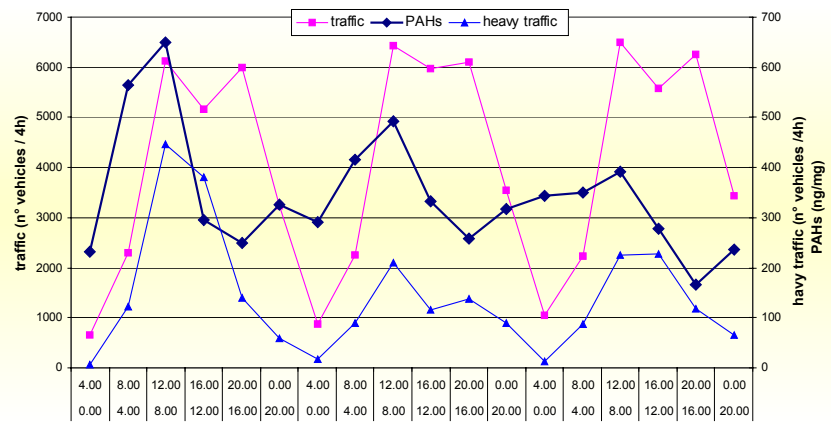

Figure 2. PAHs in 4h samples of PM2,5 (19, 20, 21 Dec 2001).

Influence of traffic on PAHs level in PM was studied considering sampling sites close to busy roads (both on the urban and extra urban area of Milan). According to a less distance of the sampling site from the traffic source, an enrichment of PAHs in PM was measured.

\section{REFERENCES}

Hanningan M.P., et.al.; "Biossay-Directed Chemical Analysis of Los Angeles Airborne Particulate Matter Using a Human Cell Mutagenicity Assay”, Env. Sci.Technol. 1998, 32, 3502

Nielsen T. et al., "Traffic contribution of polycyclic aromatic hydrocarbons in the centre of a large city"; Atmospheric Environment,20 (1996), 3481-3490

Odabasi M., et al..; "Polyciclic aromatic hydrocarbons (PAHs) in Chicago air", The Science of the total Environment 227 (1999) 57-67 